\title{
Fear Conditioning following Unilateral Temporal Lobectomy: Dissociation of Conditioned Startle Potentiation and Autonomic Learning
}

\author{
Almut I. Weike, ${ }^{1}$ Alfons 0. Hamm, ${ }^{1}$ Harald T. Schupp, ${ }^{4}$ Uwe Runge, ${ }^{2}$ Henry W. S. Schroeder, ${ }^{3}$ and Christof Kessler ${ }^{2}$ \\ Departments of ${ }^{1}$ Psychology, ${ }^{2}$ Neurology, and ${ }^{3}$ Neurosurgery, University of Greifswald, 17487 Greifswald, Germany, and ${ }^{4}$ Department of Psychology, \\ University of Konstanz, 78457 Konstanz, Germany
}

\begin{abstract}
The present study investigated fear-potentiated startle and autonomic learning in brain-lesioned patients in a classical fear-conditioning paradigm. Startle blink and skin conductance responses of 30 patients who underwent unilateral temporal lobectomy because of drugresistant epilepsy were compared with those of 32 healthy controls. As expected, temporal lobectomy patients showed a general impairment in fear conditioning relative to controls. This impairment did not differ with respect to the affected hemisphere. Moreover, while fear-conditioned startle potentiation in healthy controls was independent of contingency awareness, skin conductance discrimination was only observed for those participants who correctly recognized the stimulus contingencies. Patients who acquired a declarative memory of the contingencies also showed intact skin conductance discrimination but failed to exhibit fear-potentiated startle. The present findings support a two-levels-of-learning account of human fear conditioning and also demonstrate that the amygdala is crucially involved in fear learning.
\end{abstract}

Key words: amygdala; hippocampus; startle; skin conductance; fear conditioning; emotion

\section{Introduction}

Accumulating evidence suggests that the amygdala, a limbic structure located in the anterior medial temporal lobe, is critically involved in emotional learning and memory formation. One line of evidence comes from animal research using fear-conditioning paradigms (Fanselow, 1994; LeDoux, 1996; Davis, 2000), and sophisticated neural models about the acquisition, expression, and retention of fear have been developed (cf. Maren, 2001). Although animal experimentation is necessary to understand the neural underpinnings of affective learning, it is also important to validate these animal models in humans. Functional brainimaging studies demonstrated the involvement of the amygdala in fear conditioning in humans (Büchel and Dolan, 2000; Knight et al., 2004). This line of research is complemented by studies investigating patients with lesions of the amygdala. Bechara et al. (1995) observed a complete blocking of autonomic fear conditioning but retained declarative knowledge of the contingency between the conditioned stimulus (CS) and the unconditioned stimulus (UCS) in a patient with a confined bilateral amygdala lesion. These findings were supported by group studies revealing that patients with unilateral temporal lobectomy showed impaired conditioned skin conductance response (SCR) acquisition to aversively conditioned stimuli (LaBar et al., 1995; Peper et al.,

Received May 20, 2005; revised 0ct. 10, 2005; accepted 0ct. 16, 2005.

This work was supported by grants from the German Research Foundation (Deutsche Forschungsgemeinschaft) to A.I.W. (We 2762/3-1) and A.0.H. (Ha 1593/10-2).

Correspondence should be addressed to Dr. Almut I. Weike, Department of Psychology, University of Greifswald, Franz-Mehring-Strasse 47, 17487 Greifswald, Germany. E-mail: weike@uni-greifswald.de.

DOI:10.1523/JNEUROSCI.2032-05.2005

Copyright $\odot 2005$ Society for Neuroscience $\quad$ 0270-6474/05/2511117-08\$15.00/0
2001). Interestingly, most of these patients correctly reported the CS-UCS contingencies, thus showing acquisition of declarative memory.

Although SCR conditioning is often used to index fear learning in humans, SCRs are not specifically related to the aversiveness of stimuli but rather change with the arousal level of affective stimulation (Lang et al., 1993; Bradley, 2000), thus primarily indexing intensity of emotional stimulation. Moreover, orienting toward non-emotional but significant events also increases SCRs (cf. Öhman et al., 2000). Accordingly, increased SCRs are observed when a CS is associated with a significant but nonaversive event (Lipp et al., 1994; Hamm and Vaitl, 1996). Finally, substantial evidence indicates that conditioned SCR discrimination depends on the acquisition of declarative knowledge about the stimulus contingencies (Lovibond and Shanks, 2002), supporting the notion that SCR conditioning indexes a rather cognitive level of learning.

In contrast, the modulation of the startle reflex seems to be a more specific index for fear learning. The startle reflex is reliably potentiated during fear conditioning in both animals and humans (Hamm et al., 1993; Davis, 2000), and amygdala lesions block fear-potentiated startle in animals (Davis, 2000). In humans, conditioned startle potentiation is specifically observed for aversive UCS conditions (Hamm and Vaitl, 1996). Moreover, conditioned startle potentiation is independent of contingency learning and can even be obtained to visual cues that are not processed in the primary visual cortex (Hamm et al., 2003). Thus, the fear-potentiated startle paradigm is very useful to study the neural networks involved in emotional learning in humans.

Accordingly, the present study measured for the first time 
startle reactivity during fear conditioning in patients with unilateral removal of the amygdala. Moreover, SCRs were recorded to investigate whether the different measures of learning could help to disentangle fear conditioning and the more cognitive level of contingency learning in these patients.

\section{Materials and Methods}

Participants. Thirty-five epilepsy patients were screened for participation in the present study. The patients suffered from medically intractable unilateral temporal lobe epilepsy and had to undergo neurosurgery, which involved standardized unilateral amygdalahippocampectomy with or without resection of the anterior part of the temporal lobe. Four patients with additional lesions outside the medial temporal lobe were excluded. One additional patient had to be excluded because of equipment failure during the experiment. Of the remaining $30 \mathrm{pa}-$ tients, the resections of the medial temporal lobe (amygdalahippocampectomy) involved the left anterior part of the temporal lobe ( $4.5 \mathrm{~cm}$ from the left temporal pole) in 16 patients and the right anterior part of the temporal lobe $(5.5 \mathrm{~cm}$ from the right temporal pole) in 10 patients, respectively (Penfild and Baldwin, 1952). The remaining four patients underwent selective removals of the amygdala and hippocampus in the left $(n=2)$ or right $(n=2)$ temporal lobe, respectively (patients with selective tissue removals did not differ from patients with more extended removals in their conditioning performance or in other clinical, neuropsychological, or demographic characteristics) (Wieser and Yasargil, 1982). All patients were on low to moderate doses of anticonvulsant medication at the time of testing, which was unrelated to the patients' conditioning performance. Additional characteristics of the patients are listed in Table 1.

Thirty-two students ( 24 females) from the University of Greifswald served as a comparison group. Although the controls were overall younger than the patients $\left[F_{(1,60)}=41.64 ; p<0.001 ; \eta^{2}=0.41\right.$ (controls: 21.9 years; SE, 0.6; patients: 35.1 years; SE, 2.0)], there was a considerable range in age in both groups allowing to test whether age did affect fear conditioning. All participants signed an informed consent form before the study, which was approved by the Ethics Committee of the University of Greifswald.

Stimulus materials. Two pictures depicting male faces with neutral expressions were selected from the KDEF (Karolinska Directed Emotional Faces) (Lundqvist et al., 1998) to serve as conditioned stimuli. The pictures were projected via a slide projector (Ektapro 5000; Kodak, Stuttgart, Germany) onto a screen $2 \mathrm{~m}$ in front of the participant. The size of the visible picture was $55 \times 85 \mathrm{~cm}$. Pictures were presented for $6 \mathrm{~s}$ controlled by a tachistoscopic shutter (G1166; Gerbrands, Arlington, MA). All equipment was situated in a room adjacent to the soundshielded experimental room.

The UCS was a $10 \mathrm{~ms}$ train of single electrical pulses $(1 \mathrm{~ms})$ of $500 \mathrm{~Hz}$ generated by a commercial stimulator (S48K; Grass Instruments, West Warwick, RI). The train of pulses was isolated (SIU5; Grass Instruments) and transmitted via a constant current unit (CCU1; Grass Instruments) to a bipolar electrode (F-E10S2; Grass Instruments) at the participants' left forearm. The intensity of the UCS was adjusted individually within five warned presentations of the electrical pulse to a level that was experienced as "highly annoying, but not painful." The mean physical intensity of the UCS was comparable between the temporal lobectomy patients (8.4 mA; SE, 0.8$)$ and the control subjects ( $8.8 \mathrm{~mA}$; SE, $0.9 ; F<1)$. Within the patient group, no differences were found with regard to the affected hemisphere $(F<1)$.

The acoustic startle probe stimulus was a $50 \mathrm{~ms}$ burst of white noise with an intensity of $95 \mathrm{~dB}[\mathrm{~A}]$ (rise/fall, $<1 \mathrm{~ms}$ ) generated by a commercial noise generator (S81-02; Coulbourn Instruments, Allentown, PA) and presented binaurally over headphones (MDR-CD 170; Sony, Cologne, Germany).

Physiological recordings. Recordings of the electromyographic (EMG) activity over the left orbicularis oculi muscle served to measure the eyeblink component of the startle response. $\mathrm{Ag} / \mathrm{AgCl}$ miniature surface electrodes (Sensormedics, Yorba Linda, CA) filled with electrolyte (Marquette Hellige, Freiburg, Germany) were attached beneath the lower eyelid using adhesive rings (Marquette Hellige). The raw EMG signal was amplified and filtered through a $30-1000 \mathrm{~Hz}$ bandpass, using a commercial bioamplifier (S75-01; Coulbourn Instruments). Digital sampling with a rate of $1000 \mathrm{~Hz}$ started $100 \mathrm{~ms}$ before until $400 \mathrm{~ms}$ after the onset of the acoustic startle stimulus. The EMG signal was filtered off-line through a $60 \mathrm{~Hz}$ high-pass filter and was rectified and integrated (time constant, 10 ms) using a digital filter.

Skin conductance was recorded from the hypothenar eminence of the palmar surface of the participant's right hand. A Coulbourn Instruments S71-22 skin conductance coupler provided a constant $0.5 \mathrm{~V}$ across two $\mathrm{Ag} / \mathrm{AgCl}$ standard electrodes ( $8 \mathrm{~mm}$ diameter; Marquette Hellige) filled with a 0.05 molar sodium chloride electrolyte medium. The signal was processed with a resolution of $0.01 \mu \mathrm{S}$ and sampled with a rate of $10 \mathrm{~Hz}$. Data acquisition and stimulus presentations were synchronized using an IBM-compatible computer.

Procedure. On arrival at the laboratory, participants first read and signed an informed consent form. Then the participants were seated in a reclining chair in an upright position and were told that a number of slides would be presented during the experiment and that the acoustic stimuli being presented occasionally via headphones could be ignored. After attaching the sensors for physiological data recording, six startle probes were presented for an initial habituation of the startle eyeblink response to a stable baseline. After these initial startle probes, a series of six pictures depicting different neutral facial expressions were presented with both conditioned stimuli presented last to serve as a preconditioning baseline measure. Four of the six picture presentations were followed by an acoustic startle probe either 4.5 or $5.0 \mathrm{~s}$ after picture onset (probes were always delivered during the designated CS + and CS - ). Two startle probes were presented during the intertrial intervals (ITIs) that varied between 14 and $21 \mathrm{~s}$.

After this preconditioning phase, the experimenter entered the room and attached the electrodes for electric stimulation to the participant's left forearm. The intensity of the electric pulse was adjusted within five warned presentations of the pulse to a level that the participant described as highly annoying, but not painful. Participants were instructed that during the following phase of the experiment, a series of slides and some electric pulses would be presented and that the acoustic stimuli being presented occasionally via headphones could be ignored. No informa- 
tion on the contingencies between the slides and the electric pulses was provided.

The following differential delay conditioning phase consisted of nine presentations of each CS [i.e., one of the neutral male faces (CS+) always coterminated with the presentation of the electric UCS, whereas the other face (CS-) was never paired with the UCS]. The order of presentation of the conditioned stimuli was perceptually random with the restriction of no more than two consecutive presentations of the same picture. In six of nine presentations of each CS, an acoustic startle probe was administered at either 4.5 or $5.0 \mathrm{~s}$ after CS onset (three times each). Six acoustic startle probes were presented during the ITIs that varied between 14 and $22 \mathrm{~s}$. The conditioning phase was immediately followed by the postconditioning trials (i.e., neither a break nor any signal indicated that no additional uconditioned stimuli would be presented). The postconditioning phase consisted of nine presentations of each CS. The order of CS presentation and the timing of the startle probes was the same as during the previous conditioning phase.

After the postconditioning phase, a postexperimental interview was performed to determine whether the subjects were aware of the CS-UCS contingency (Dawson and Schell, 1987; Bechara et al., 1995). First, a "free recall" question served to determine the subject's ability to verbalize the CS-UCS contingency ("Did you know when you were going to receive the electrotactile stimulus?"). Then the subject was asked how many different faces he/she had seen during the experiment and a description of each of the faces was requested. Several additional questions addressed possible differences between the faces and whether there might have been a relationship between the presentation of the faces and the electrotactile stimulus. The interview ended with a final multiple choice "recognition" question: "The presentation of an electrotactile stimulus (UCS) followed: (1) the male face with... (individual description), (2) the male face with... (individual description), (3) there was no systematic relationship, or (4) not possible to answer."

Data reduction and response definition. The magnitude of the startle blink was scored off-line using a computer program (Globisch et al., 1993) that identified latency of the blink onset (in milliseconds) and peak amplitude (in microvolts). Responses starting 20-100 ms after startle probe onset and reaching peak amplitude within $150 \mathrm{~ms}$ from probe onset were identified as startle blinks. For trials in which no response could be detected, the magnitude was scored as zero. Trials with excessive baseline activity or recording artifacts were rejected. Overall, 45 of 2976 trials $(1.5 \%)$ had to be discarded. The proportion of missing trials was higher in the patient group $(2.3 \%)$ than the controls $(0.8 \%)\left(F_{(1,60)}=\right.$ $\left.7.9 ; p<0.05 ; \eta^{2}=0.12\right)$, but the number of missing trials per subject did not exceed five trials. Before the statistical data analyses, all missing values were replaced individually for each participant by the overall mean blink response magnitude of all 48 trials in which startle probes were presented (regardless of the experimental condition) as a conservative estimate of the response magnitude. The overall mean startle blink magnitude was higher in the control subjects $(M=81.2 \mu \mathrm{V}$; SE, 12.2) than in the patients $(M=26.7 \mu \mathrm{V} ; \mathrm{SE}, 3.6)\left(F_{(1,60)}=17.4 ; p<0.05 ; \eta^{2}=0.23\right)$. To control for this interindividual variability, startle blink magnitude data were standardized. The responses of each participant were converted to $T$ scores [i.e., $50+(z \times 10)]$.

SCR magnitudes to the conditioned stimuli were scored as the largest increase in conductance between 0.9 and $4.0 \mathrm{~s}$ after picture onset (first interval response) (Prokasy and Kumpfer, 1973) using a computer program (Globisch et al., 1993). These responses were not confounded by the presentation of the startle probe stimuli. The unconditioned response was scored as the largest increase in conductance between 0.9 and $4.0 \mathrm{~s}$ after the onset of the electrical stimulus. Trials in which no response could be detected were considered as zero responses. Trials with respiration or recording artifacts were rejected. Overall, 122 of 3472 trials $(3.5 \%)$ had to be discarded. The proportion of missing trials did not differ between patients $(3.5 \%)$ and controls $(3.6 \% ; F<1)$. Missing trials were replaced individually for each participant by the mean response magnitude to all picture trials (averaged across CS + and CS - presentations) or the mean unconditioned response magnitude averaged across all UCS presentations, respectively. Logarithms of all values were computed before statistical analyses to normalize the distribution (Venables and Christie, 1980). To reduce interindividual variability that was not related to the conditioning task, the log values were range corrected by dividing each individual score by the participant's maximum response (Lykken and Venables, 1971).

Data analysis. Physiological data were analyzed separately for each phase of the experiment. Mixed-model ANOVAs were calculated for each phase for startle blink and SCR magnitudes, respectively. These ANOVAs involved conditioning (CS + vs CS - vs ITI for startle blink data and CS + vs CS - for skin conductance data, respectively) and trialblock [early (trials 1-3) vs middle (trials 4-6) vs late (trials 7-9)] as the within-subject factors and group (patients vs controls) as a betweensubjects factor. [For evaluation of conditioned startle blink responses, the blink magnitudes during the ITI need to be included in the analyses, because startle potentiation (CS + vs ITI) is considered the core measure of affective learning and can be more easily related to animal data. Thus, the overall analyses were followed by post hoc tests involving startle potentiation (CS+ vs ITI) and startle discrimination (CS+ vs CS-).] After these analyses, comparisons were made within each group. Mixed-model ANOVAs were calculated for the patient group involving the withinsubjects factors defined above and the between-subject factor lesion site (left vs right). Because of the age-related differences between the patients and controls, all analyses involving group differences were calculated again with age entered as a covariate in these analyses. These analyses of covariance results are only reported if they revealed significant effects. Unless noted otherwise, all statistical tests used the 0.05 level of statistical significance. Degrees of freedom were adjusted when appropriate using the Greenhouse-Geisser $\epsilon$ correction. Nominal degrees of freedom are reported along with $\epsilon$ values. Partial $\eta^{2}$ values (or $w$ for a $\chi^{2}$ test) are reported as a measure of effect size.

\section{Results}

\section{Preconditioning}

Startle blink response magnitudes

Before conditioning, blink responses elicited during viewing of the conditioned stimuli were significantly smaller than those elicited without any visual foreground $\left(F_{(2,120)}=7.39 ; p<0.01 ; \epsilon=\right.$ $\left.0.96 ; \eta^{2}=0.11\right)$, whereas startle blink magnitudes did not differ between the designated CS + and $\mathrm{CS}-(F<1)$. No differences were observed between the patients and the control subjects $(F<$ 1). Moreover, this relative startle inhibition before the conditioning tended to be more pronounced in patients with right compared with left-hemisphere lesions $\left(F_{(2,56)}=2.53 ; p=0.096\right.$; $\left.\eta^{2}=0.08\right)$.

\section{SCR magnitudes}

As expected, SCRs to the CS + and CS - did not differ before conditioning $(F<1)$ and were also not different between patients and controls $(F<1)$. SCRs also did not differ between the patients with respect to the lesion site $(F<1)$.

\section{Responses to the UCS during shock exposure}

The UCS adjustment procedure resulted in comparable UCS intensity in patients and controls, respectively. Accordingly, the unconditioned SCR magnitudes elicited by the UCS were comparable across patients $(0.51 \mu \mathrm{S} ; \mathrm{SE}, 0.12)$ and controls $(0.80 \mu \mathrm{S}$; $\mathrm{SE}, 0.12)\left(F_{(1,60)}=2.94 ; p=0.091 ; \eta^{2}=0.05\right)$. Moreover, no differences between the patients' UCR magnitudes were observed with respect to the affected hemisphere $(F<1)$.

\section{Sensitization after UCS exposure}

\section{Startle blink response magnitudes}

Replicating previous findings, a pronounced sensitization of the startle reflex was observed after repeated electric shock exposure $\left(F_{(1,60)}=66.47 ; p<0.001 ; \eta^{2}=0.53\right)$, which did not differ between patients $(M=16.7$; SE, 2.6) and controls $(M=14.4$; SE, $2.8 ; F<1)$ [shock sensitization effects were analyzed comparing 
the physiological responses to the last CS presented before the shock work-up procedure (regardless of whether this was the designated $\mathrm{CS}+$ or $\mathrm{CS}-$ ) with those elicited during the first CS presentation during the conditioning phase (again regardless whether this was the CS+ or the CS -, because no CS has been paired with a UCS at this time)]. Moreover, startle sensitization was equally pronounced in patients with left- or righthemisphere lesions $(F<1)$.

\section{SCR magnitudes}

As was the case for startle blink magnitudes, UCS exposure resulted in a pronounced increase of SCR magnitudes to the first CS presentation in the conditioning phase $\left(F_{(1,60)}=31.97 ; p<\right.$ $\left.0.001 ; \eta^{2}=0.35\right)$. This sensitization effect again did not differ between the patients $(M=0.13$; SE, 0.04$)$ and the control subjects $(M=0.17$; SE, $0.03 ; F<1)$, suggesting that the UCS was effective in both groups of participants. Moreover, the sensitization was not affected by lesion site $(F<1)$.

\section{Conditioning}

Startle blink response magnitudes

Replicating previous findings, blink magnitudes elicited during the presentation of the CS + were significantly potentiated relative to the blink responses elicited during the ITI $\left(F_{(1,60)}=11.44\right.$; $\left.p<0.01 ; \eta^{2}=0.16\right)$ or compared with those elicited during the $\mathrm{CS}-\left(F_{(1,60)}=11.84 ; p<0.01 ; \eta^{2}=0.17\right)$, resulting in a significant main effect of conditioning $\left(F_{(2,120)}=8.58\right.$; $p<0.001 ; \epsilon=$ $0.94 ; \eta^{2}=0.13$ ) (Fig. 1). As expected, this conditioned startle potentiation was impaired in patients relative to controls (conditioning by group: $\left.F_{(2,120)}=3.20 ; p<0.05 ; \epsilon=0.94 ; \eta^{2}=0.05\right)$. While the controls exhibited a pronounced fear-potentiated startle blink response $\left(F_{(2,62)}=8.83 ; p<0.01 ; \epsilon=0.92 ; \eta^{2}=0.22\right)$, no significant modulation of the startle blink response was observed in the patient group $\left(F_{(2,58)}=2.58 ; p=0.088 ; \epsilon=0.95\right.$; $\left.\eta^{2}=0.08\right)$. Post hoc analyses revealed that while control subjects exhibited significantly larger blink magnitudes during $\mathrm{CS}+$ relative to the ITI $\left(F_{(1,31)}=13.4 ; p<0.01 ; \eta^{2}=0.30\right)$, no such startle potentiation was observed for the patients $(F<1)$. This effect was supported by a significant potentiation-by-group interaction $\left(F_{(1,60)}=5.00 ; p<0.05 ; \eta^{2}=0.08\right)$. This interaction was not significant for the conditioned startle discrimination $(F<1)$ [i.e., startle blink responses during the $\mathrm{CS}+$ were larger than during the $\mathrm{CS}-$ for both patients $\left(F_{(1,29)}=4.27 ; p<0.05 ; \eta^{2}=0.13\right)$ and controls $\left.\left(F_{(1,31)}=7.88 ; p<0.01 ; \eta^{2}=0.20\right)\right]$. No differences in startle blink responding were observed between patients with left- or right-hemisphere lesions, respectively $(F<1)$.

\section{SCR magnitudes}

As expected, SCRs to the CS + were overall larger than those to the CS $-\left(F_{(1,60)}=19.77 ; p<0.001 ; \eta^{2}=0.25\right)$. Replicating previous findings, this SCR conditioning differed significantly between patients and controls (conditioning by group: $F_{(1,60)}=$ 8.04; $p<0.01 ; \eta^{2}=0.12$ ). While the control group exhibited robust differential conditioning $\left(F_{(1,31)}=21.78 ; p<0.001 ; \eta^{2}=\right.$ $0.41)$, electrodermal responses to the CS + and CS - did not differ in the patient group $\left(F_{(1,28)}=1.60 ; p=0.216 ; \eta^{2}=0.08\right)$ (Fig. $2)$. This impaired skin conductance conditioning in the patients was not modulated by the site of the lesion $(F<1)$.

\section{Postconditioning}

Startle blink response magnitudes

Startle eyeblinks elicited during the CS + continued to be potentiated both relative to the ITI $\left(F_{(1,60)}=10.28 ; p<0.01 ; \eta^{2}=\right.$ $0.15)$ and relative to the $\mathrm{CS}-\left(F_{(1,60)}=5.47 ; p<0.05 ; \eta^{2}=0.08\right)$,
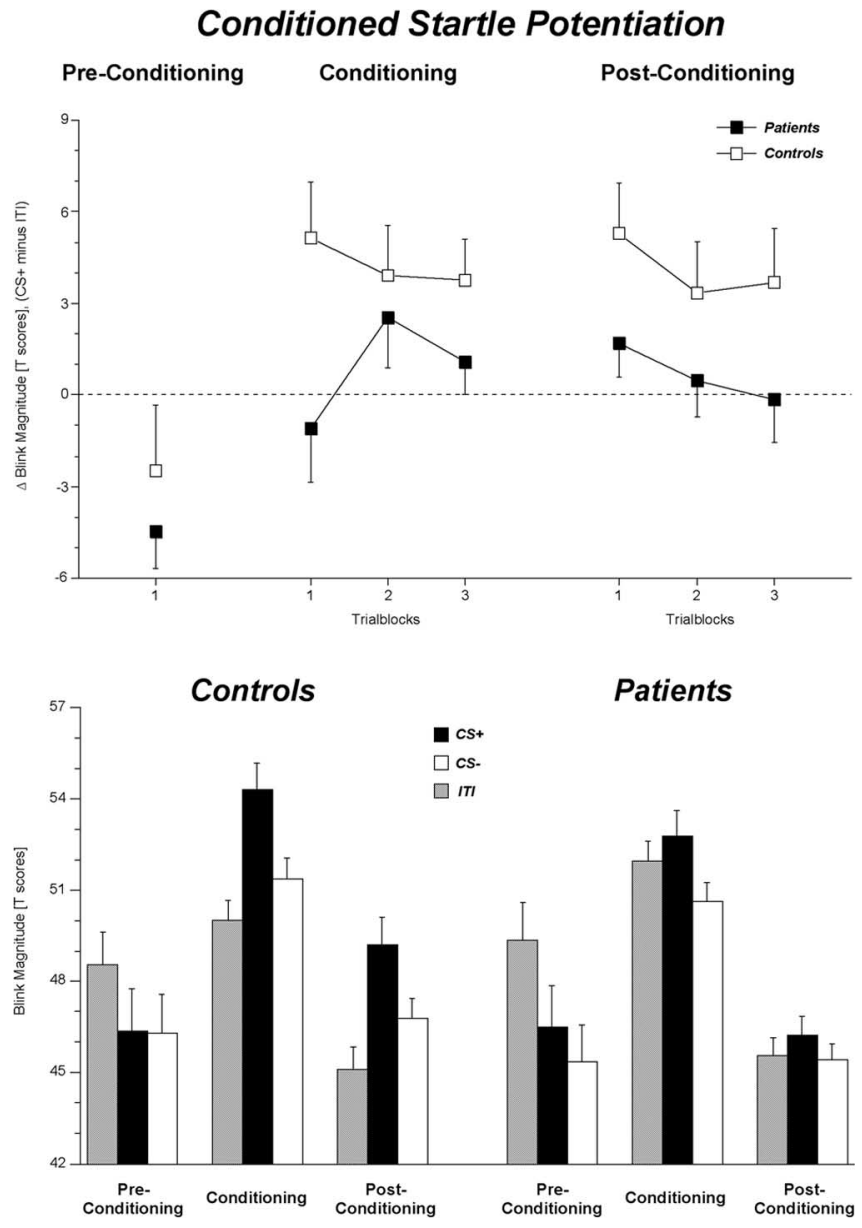

Figure 1. Mean $( \pm S E)$ conditioned startle blink response potentiation for patients and control subjects before, during, and after the classical fear conditioning procedure. Difference scores are illustrated in the top panel referring to the conditioned potentiation (CS + minus ITI), whereas the bottom panel depicts mean startle blink magnitudes elicited during the $\mathrm{CS}+$, CS- , and ITI.

\section{Differential SCR Conditioning}

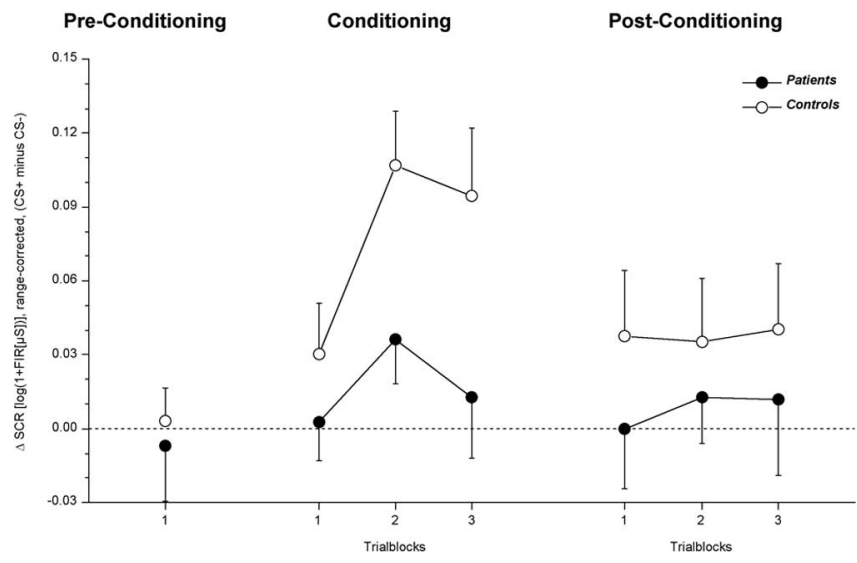

Figure 2. Mean ( \pm SE) SCR discrimination (CS + minus $(S-)$ for patients $(-)$ and control subjects $(\bigcirc)$ before, during, and after the differential conditioning.

as reflected in a significant main effect of conditioning $\left(F_{(2,120)}=\right.$ 6.45; $\left.p<0.01 ; \epsilon=0.94 ; \eta^{2}=0.10\right)$. Again, this conditioned startle potentiation was impaired in the patient group during postconditioning (conditioning by group: $F_{(2,120)}=3.22 ; p<$ 
0.05; $\epsilon=0.94 ; \eta^{2}=0.05$ ) (Fig. 1). Similar to the conditioning phase, control subjects showed a pronounced conditioned startle potentiation (conditioning: $F_{(2,62)}=6.89 ; p<0.01 ; \epsilon=0.92$; $\eta^{2}=0.18$ ), whereas the patients' blink responses did not differ among the CS+, the CS-, and the ITI (conditioning; $F<1$ ). Again, no differences were observed between patients with leftor right-hemisphere lesions $(F<1)$. Post hoc analyses revealed that the patients' startle blink responses elicited during the CS+ neither differed from those elicited during the CS $-\left(F_{(1,29)}=\right.$ $\left.1.30 ; p=0.263 ; \eta^{2}=0.04\right)$ nor from those elicited during the ITI $(F<1)$. In contrast, in the control group, both conditioned startle potentiation $\left(\mathrm{CS}+\right.$ vs ITI; $F_{(1,31)}=11.38 ; p<0.01 ; \eta^{2}=$ 0.27 ) as well as conditioned startle discrimination (CS + vs CS-; $F_{(1,31)}=4.35 ; p<0.05 ; \eta^{2}=0.12$ ) were still observed during the postconditioning.

\section{SCR magnitudes}

As illustrated in Figure 2, the CS + continued to elicit larger SCRs relative to the CS - in the control group $\left(F_{(1,31)}=4.46 ; p<0.05\right.$; $\left.\eta^{2}=0.13\right)$, whereas no SCR discrimination was observed in the patients $(F<1)$, although the interaction fell short of statistical significance (conditioning by group: $F_{(1,60)}=1.27 ; p=0.265$; $\eta^{2}=0.02$ ). [During postconditioning, the control subjects' SCRs to the first CS - after the first unreinforced CS+ significantly increased relative to the SCRs to the last CS- during the conditioning phase $\left(M_{\text {pre }}=0.05 \mu \mathrm{S} ; M_{\text {post }}=0.12 \mu \mathrm{S} ; F_{(1,31)}=5.86\right.$; $p<0.05)$ as a result of contingency reversal expectation. This increase in SCRs to CS - resulted in a lack of response discrimination during the very first trial of postconditioning. Therefore, the first postconditioning trial was excluded from further analyses in this variable.] Furthermore, no effects of lesion site could be observed within the patients $\left(F_{(1,28)}=2.03 ; p=0.165 ; \eta^{2}=\right.$ $0.07)$.

\section{Postexperimental evaluation of declarative knowledge}

While $75 \%$ of the control subjects ( 24 of 32 ) were aware of the CS-UCS contingency after the experiment, only $30 \%$ of the patients $(9$ of 30$)$ were able to correctly identify the CS $+\left(\chi_{(1,62)}^{2}=\right.$ $12.59 ; p<0.001 ; w=0.45$ ). (Contingency awareness was evaluated by the final multiple choice question at the end of the postexperimental interview, because the free recall question underestimated the ratio of aware subjects. Only 3 patients and 15 controls were able to correctly report the CS-UCS contingencies in the recall test, whereas an additional 6 patients and 9 controls were able to correctly choose the CS+ in the multiple choice question.) Moreover, the patients' ability to recognize the stimulus contingency was not associated with the affected hemisphere $\left(\chi_{(1,30)}^{2}=1.30 ; p=0.255 ; w=0.21\right)$ (i.e., of nine aware patients, four patients had left-hemisphere and five patients had righthemisphere lesions). No systematic relationships between contingency awareness and the patients' neuropsychological performance or clinical characteristics were observed (note that a more comprehensive neuropsychological assessment might have revealed some differences in explicit memory performance between aware and unaware patients). Further analyses served to explore the role of awareness of the stimulus contingencies on the psychophysiological measures of fear learning during conditioning.

Replicating previous findings, the conditioned startle potentiation did not differ for aware and unaware subjects. As expected, startle potentiation was equally pronounced in aware and unaware controls (conditioning: $F_{(2,60)}=5.84, p<0.01, \epsilon=$ $0.91, \eta^{2}=0.16$; conditioning by awareness: $F<1$ ) (Fig. 3, top).
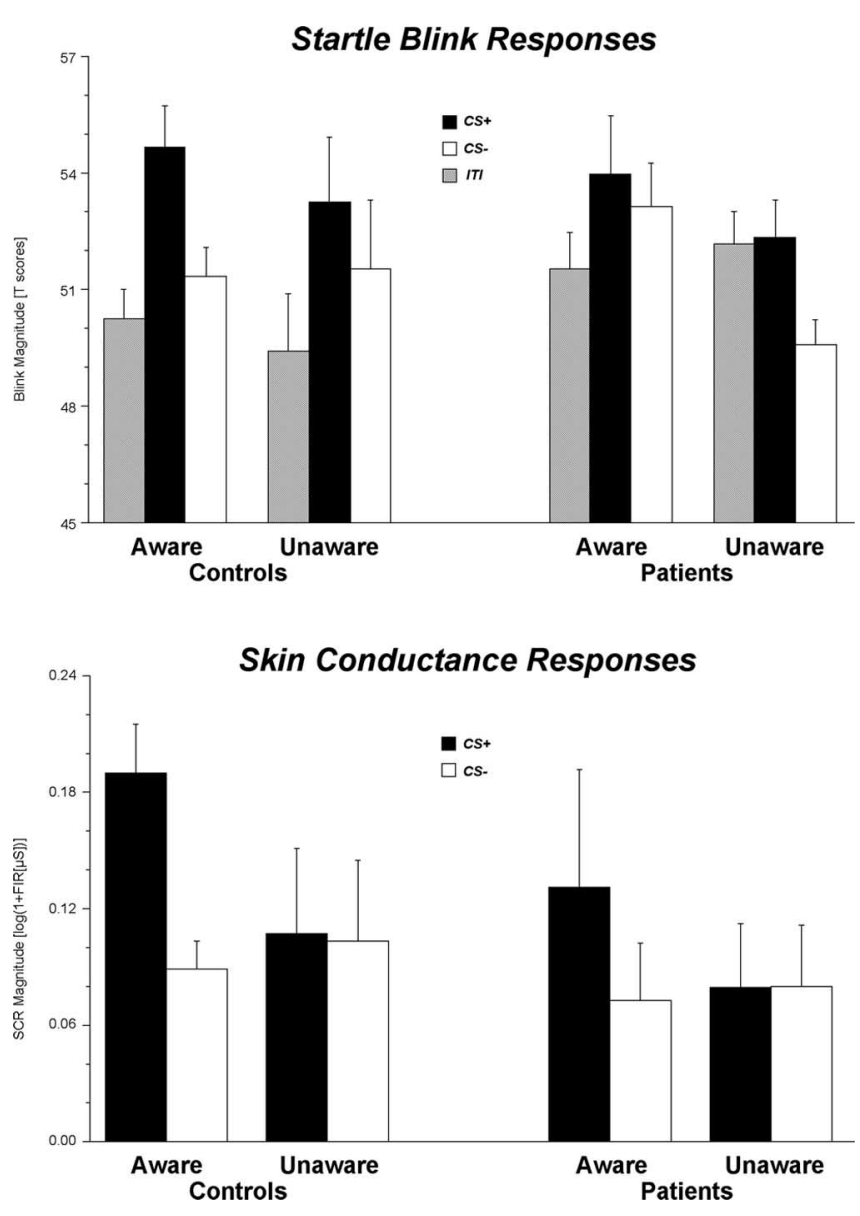

Figure 3. Mean ( $\pm \mathrm{SE}$ ) startle blink response (top) and mean SCR (bottom) magnitudes during the conditioning phase for control subjects and patients, who were either aware or unaware of the CS-UCS contingencies at the end of the experiment. Note that although visual inspection of the top panel suggests an interaction between the conditioned startle modulation effects and the patients' awareness, neither effect was found to be statistically reliable, whereas electrodermal conditioning (bottom panel) differed for aware and unaware patients.

Blink magnitudes were significantly larger during CS + both relative to ITI $\left(F_{(1,30)}=9.06 ; p<0.01 ; \eta^{2}=0.32\right)$ and relative to $\mathrm{CS}-\left(F_{(1,30)}=4.32 ; p<0.05 ; \eta^{2}=0.13\right)$. Neither of these significant effects was modulated by awareness $(F<1)$. In addition, the impaired conditioned startle potentiation found in the patient group $\left(F_{(2,56)}=1.60 ; p=0.214 ; \epsilon=0.91 ; \eta^{2}=0.05\right)$ also did not vary as a function of contingency awareness (conditioning by awareness: $F_{(2,56)}=2.07 ; p=0.141 ; \epsilon=0.91 ; \eta^{2}=0.07$ ). Thus, although Figure 3 (top) suggests an interaction between the conditioned startle modulation effects and the patients' awareness, neither effect was found to be statistically reliable [i.e., neither startle potentiation (CS + vs ITI) nor startle discrimination $(\mathrm{CS}+$ vs $\mathrm{CS}-$ ) differed between aware and unaware patients $\left(\mathrm{CS}+\right.$ vs ITI: $F_{(1,28)}=1.15, p=0.293, \eta^{2}=0.04$; CS + vs CS-: $\left.\left.F_{(1,28)}=2.40, p=0.132, \eta^{2}=0.08\right)\right]$.

In contrast to conditioned startle potentiation, the conditioned SCRs differed for aware and unaware subjects. Replicating previous results, differential electrodermal conditioning was only observed for those subjects who were aware of the CS-UCS contingencies (conditioning by awareness: $F_{(1,58)}=12.75 ; p<0.01$; $\eta^{2}=0.20$ ) (Fig. 3, bottom). Moreover, the conditioning-byawareness-by-group interaction was not significant $(F<1)$, suggesting that contingency awareness similarly modulated SCR conditioning in patients and controls. However, additional anal- 
yses were calculated to ensure that these results were not attributable to the unbalanced ratio of aware and unaware patients and controls. As expected, the electrodermal conditioning effects differed between aware and unaware controls [conditioning by awareness: $\left.F_{(1,30)}=7.92, p<0.01, \eta^{2}=0.21\right]$. Only those controls who memorized the stimulus contingencies exhibited the typical pattern of increasing response discrimination during the conditioning phase [conditioning by trialblock: $F_{(2,46)}=3.82$, $\left.p<0.05, \epsilon=0.770, \eta^{2}=0.14\right]$, whereas SCR magnitudes did not differ between the CS+ and CS - in unaware controls $(F<1)$. More importantly, similar associations between the declarative knowledge and the electrodermal conditioning were also observed for the patients (conditioning by awareness: $F_{(1,28)}=4.85$; $p<0.05 ; \eta^{2}=0.15$ ). Additional analyses indicated that the CS+ elicited increasingly larger SCRs than the CS- during the conditioning in those patients, who were able to correctly recognize the stimulus contingencies after the experiment (conditioning by trialblock: $\left.F_{(2,16)}=3.14 ; p=0.085 ; \epsilon=0.815 ; \eta^{2}=0.28\right)$, whereas no such effects could be observed for the unaware patients $(F<$ $1)$.

Additional correlational analyses were undertaken to examine the general relationship between startle and SCR measures of aversive learning. As expected, the amount of conditioned startle potentiation (CS + minus ITI) was correlated to the amount of conditioned startle discrimination ( $\mathrm{CS}+$ minus $\mathrm{CS}-$ ) in controls $\left(r_{\mathrm{s}}=0.61 ; p<0.001\right)$ and patients $\left(r_{\mathrm{s}}=0.58 ; p<0.01\right)$. In contrast, no relationship was observed between the startle measures and the conditioned skin conductance responding, supporting the notion that both physiological response systems reflect different aspects of fear learning. Specifically, the amount of SCR conditioning was unrelated to the amount of startle potentiation and startle discrimination in the patient group $\left(r_{\mathrm{s}}=0.14\right.$ and $0.26 ; p>0.172)$ and in the control group $\left(r_{\mathrm{s}}=0.02\right.$ and $-0.03 ; p>0.883)$, respectively. Although the correlation coefficients might suggest a trend toward significance in the patient group, this was mainly attributable to one outlier. Reanalyses excluding this outlier confirmed that neither the amount of startle potentiation nor startle discrimination was correlated with the amount of conditioned SCR $\left(r_{\mathrm{s}}=0.05\right.$ and $\left.0.18 ; p>0.364\right)$, whereas both startle measures remained to be highly related to each other $\left(r_{\mathrm{s}}=0.53 ; p<0.01\right)$.

\section{Discussion}

The present study assessed conditioned startle potentiation and autonomic learning in temporal lobectomy patients and healthy controls using a classical fear-conditioning paradigm. Conditioned startle potentiation was clearly impaired in patients with unilateral lesions of the amygdala compared with healthy controls. These findings support animal data that have consistently shown that lesions of the amygdala abolish fear-potentiated startle in rodents (Koch, 1999; Walker and Davis, 2002). The present results are also in line with recent research revealing impaired augmentation of the startle reflex in patients with temporal lobe damage during viewing of highly arousing unpleasant pictures (Funayama et al., 2001; Buchanan et al., 2004). Together, these data demonstrate that the amygdaloid complex is critically involved in startle reflex potentiation by aversive and threatening stimulation.

Moreover, replicating previous findings (LaBar et al., 1995; Peper et al., 2001), there was also a clear impairment in skin conductance learning in patients after unilateral temporal lobectomy compared with healthy controls. However, in contrast to conditioned startle potentiation, SCR conditioning was closely related to the cognitive representation or declarative knowledge of the stimulus contingencies. Replicating previous research (Hamm and Vaitl, 1996), SCRs to the reinforced CS were only larger for those control subjects who correctly memorized the CS-UCS contingencies. Importantly, the same effect could be observed for temporal lobectomy patients. Those patients who acquired declarative knowledge of the stimulus contingencies also exhibited intact autonomic learning despite the unilateral removal of the amygdala. Interestingly, although these patients learned the contingencies (i.e., they knew on a cognitive level that the CS + predicted the occurrence of the UCS), they did not show a conditioned fear response: no larger startle responses were observed during the CS+ than during the CS - or the ITI in these patients, thus further supporting the hypothesis that the amygdala is crucially involved in the transmission of the emotional salience of the CS.

Although the close relationship between contingency awareness and skin conductance discrimination is in line with recent findings by Grillon (2002), no conditioned startle potentiation for healthy unaware participants was observed in this fearconditioning study, which is at odds with the current results. Some procedural differences might be responsible for these discrepant results. While different faces served as conditioned stimuli in the present study, Grillon (2002) used pictures of flowers and mushrooms, stimuli that showed poorer conditioning effects in a series of previous studies in preparedness research (Öhman and Mineka, 2001). Moreover, in the study by Grillon (2002), statistical analyses used raw blink magnitudes that are highly susceptible to individual outliers, whereas standardized scores were used for all dependent variables in the current experiment to ensure that each participant equally contributes to the group mean.

It is noteworthy that $75 \%$ of the control subjects but only $30 \%$ of the temporal lobectomy patients were able to correctly report the CS-UCS relationship in the present study. The rather high proportion of patients who failed to pick up the stimulus contingencies contrasts to the findings of LaBar et al. (1995). In this study, almost all patients demonstrated contingency awareness. Several issues might be relevant for these differences. There is ample evidence that the hippocampal formation is critically involved in contingency learning and declarative memory (Bechara et al., 1995; Clark and Squire, 1998; Eichenbaum, 2004). Thus, the different proportions of aware patients across studies may reflect more extended lesions of the temporal lobe in the present sample. Actually, the neurosurgery involved tissue removals of $4.5 \mathrm{~cm}$ from the left or $5.5 \mathrm{~cm}$ from the right temporal pole compared with $3.5 \mathrm{~cm}$ resections in the sample of LaBar et al. (1995). In addition, patients with right temporal lobectomy scored slightly below average in the verbal declarative memory task, suggesting that the present patient group had a more restricted explicit memory status relative to those studied by LaBar et al. (1995). Moreover, while different faces served as conditioned stimuli in the current experiment, pure tones were used in the study by LaBar et al. (1995). Facial stimuli are preferentially processed in distinct brain regions, and a number of studies revealed that emotion recognition from facial stimuli is impaired in patients with amygdala lesions (Adolphs, 2002). However, neutral facial expressions were used in the present study; thus, the basic processing of these stimuli should not have been affected by the patients' lesions. Finally, an on-line measure of awareness might have revealed larger proportions of aware patients. However, because on-line measures increase task demands and might interfere with conditioning, we used a postexperimental measure 
being as conservative as possible in considering participants as unaware. Given the present finding of a dissociation of startle and SCR measures during fear conditioning, it appears reasonable to assume that these results were not attributable to some misclassified unaware participants.

The observed impairments of fear-potentiated startle and SCR conditioning in the patients were independent of lesion site, which is in line with findings from La Bar et al. (1995), who also did not find differences between the right and left temporal lobectomy groups in autonomic fear conditioning. In contrast, $\mathrm{Fu}-$ nayama et al. (2001) found a remarkable dissociation of affective startle modulation and lesion site, depending on the task involved. Patients with right temporal lesions showed intact startle potentiation when instructed that one of two stimuli might be followed by an aversive event (instructed fear paradigm) (Grillon and Davis, 1995), whereas they failed to exhibit the typical augmentation of the startle reflex during viewing of unpleasant images. Patients with left temporal lesions showed the reversed pattern of results (i.e., intact startle potentiation during viewing of unpleasant images but impaired startle potentiation in the instructed fear paradigm). In a similar vein, a recent study by Gläscher and Adolphs (2003) revealed that patients with left amygdala damage showed an impaired covariation between the rated emotional intensity and the SCRs to affective pictures, whereas patients with right amygdala damage showed an overall impairment of autonomic responding. Lateralized activations of the amygdaloid complex have also been observed in neuroimaging studies in healthy participants. Consistent with the patient study by $\mathrm{Fu}-$ nayama et al. (2001), the instructed fear paradigm involved selective activations of the left amygdala in healthy volunteers (Phelps et al., 2001). Moreover, briefly presented, unmasked conditioned stimuli involved activations of the left amygdala, whereas right amygdala activations have been observed for masked conditioned stimuli (Morris et al., 1998). In the current study, the conditioned stimuli were presented unmasked, suggesting a preferential involvement of the left amygdala. In contrast, the CS also becomes a threatening stimulus by direct experience, a learning experience known to activate the right amygdala (Dolan and Morris, 2000). Therefore, both processes might have overshadowed potential laterality effects in the current study.

In the present patient sample, the extent of the amygdalahippocampetomy differed between the four patients who underwent a selective removal and the remaining patients with additional resections of the anterior temporal lobe. Thus, other temporal lobe structures than the amygdala and hippocampus might be involved in the present findings. However, no differences in fear conditioning were observed between patients with selective or extended lesions in the current study, although the small sample size did not allow for a conclusive statistical evaluation of the possible effects of lesion size on fear conditioning.

In conclusion, the current data clearly revealed a dissociation between fear learning as indexed by conditioned startle potentiation and the learning of stimulus relationships as indexed by conditioned SCR discrimination, thus supporting a two-level account of human fear conditioning (Hamm and Weike, 2005; Öhman and Mineka, 2001). In a typical human fear conditioning experiment, the subjects not only learn to fear the CS (emotional learning) (LeDoux, 1996) but they also learn on a cognitive level that one CS predicts the occurrence of the UCS [contingency learning (Rescorla, 1988); propositional learning (Lovibond and Shanks, 2002)]. Differential SCR conditioning was associated with the acquisition of declarative knowledge about the stimulus contingencies: those patients with unilateral amygdala lesions who correctly recognized the CS-UCS contingencies also showed clear evidence of skin conductance learning. Nonetheless, these patients failed to exhibit conditioned startle potentiation. That is, both levels of learning were unrelated to each other as was further substantiated by the correlation analyses revealing a lack of covariation between the amounts of fear potentiated startle and SCR conditioning in both patients and controls. Thus, the present data support findings from several neuropsychological and neurocognitive studies demonstrating the existence of multiple memory systems in the brain (Squire and Knowlton, 2000). Fear-conditioning memories as indexed by the conditioned startle potentiation appear as an implicit memory that can be activated without explicit recollection of the stimulus environment but is dependent on the functional integrity of the amygdala. In contrast, conditioned SCR discrimination seems to primarily index the learning of the rules or circumstances in which a specific stimulus is signaling a threatening event, which is a declarative and explicit memory that more likely involves activation of neural circuits centered on the hippocampal formation.

\section{References}

Adolphs R (2002) Recognizing emotion from facial expressions: psychological and neurological mechanisms. Behav Cogn Neurosci Rev 1:21-61.

Bechara A, Tranel D, Damasio H, Adolphs R, Rockland C, Damasio AR (1995) Double dissociation of conditioning and declarative knowledge relative to the amygdala and hippocampus in humans. Science 269:1115-1118.

Bradley MM (2000) Emotion and motivation. In: Handbook of psychophysiology (Cacioppo JT, Tassinary LG, Berntson GG, eds), pp 602-642. New York: Cambridge UP.

Buchanan TW, Tranel D, Adolphs R (2004) Anteromedial temporal lobe damage blocks startle modulation by fear and disgust. Behav Neurosci 118:429-437.

Büchel C, Dolan RJ (2000) Classical fear conditioning in functional neuroimaging. Curr Opin Neurobiol 10:219-223.

Clark RE, Squire LR (1998) Classical conditioning and brain systems: the role of awareness. Science 280:77-81.

Davis M (2000) The role of the amygdala in conditioned and unconditioned fear and anxiety. In: The amygdala, Vol 2 (Aggleton JP, ed), pp 213-287. Oxford: Oxford UP.

Dawson ME, Schell A (1987) Human autonomic and skeletal classical conditioning: the role of conscious cognitive factors. In: Cognitive processes and Pavlovian conditioning in humans (Davey G, ed), pp 27-55. New York: Wiley.

Dolan RJ, Morris JS (2000) The functional anatomy of innate and acquired fear: perspectives from neuroimaging. In: Cognitive neuroscience of emotion (Lane RD, Nadel L, eds), pp 225-241. New York: Oxford UP.

Eichenbaum H (2004) Hippocampus: cognitive processes and neural representations that underlie declarative memory. Neuron 44:109-120.

Fanselow MS (1994) Neural organization of the defensive behavior system responsible for fear. Psychon Bull Rev 1:429-438.

Funayama ES, Grillon C, Davis M, Phelps EA (2001) A double dissociation in the affective modulation of startle in humans: effects of unilateral temporal lobectomy. J Cogn Neurosci 13:721-729.

Gläscher J, Adolphs R (2003) Processing of the arousal of subliminal and supraliminal emotional stimuli by the human amygdala. J Neurosci 23:10274-10282.

Globisch J, Hamm AO, Schneider R, Vaitl D (1993) A computer program for scoring reflex eyeblink and electrodermal responses written in PASCAL. Psychophysiology 30 [Suppl]:S30.

Grillon C (2002) Associative learning deficits increase symptoms of anxiety in humans. Biol Psychiatry 51:851-858.

Grillon C, Davis M (1995) Acoustic startle and anticipatory anxiety in humans: effects of monaural right and left ear stimulation. Psychophysiology 32:155-161.

Hamm AO, Vaitl D (1996) Affective learning: awareness and aversion. Psychophysiology 33:698-710.

Hamm AO, Weike AI (2005) The neuropsychology of fear learning and fear regulation. Int J Psychophysiol 57:5-14. 
Hamm AO, Greenwald MK, Bradley MM, Lang PJ (1993) Emotional learning, hedonic change, and the startle probe. J Abnorm Psychol 102:453-465.

Hamm AO, Weike AI, Schupp HT, Treig T, Dressel A, Kessler C (2003) Affective blindsight: intact fear conditioning to a visual cue in a cortically blind patient. Brain 126:267-275.

Knight DC, Smith CN, Cheng DT, Stein EA, Helmstetter FJ (2004) Amygdala and hippocampal activity during acquisition and extinction of human fear conditioning. Cogn Affect Behav Neurosci 4:317-325.

Koch M (1999) The neurobiology of startle. Progr Neurobiol 59:107-128.

LaBar KS, LeDoux JE, Spencer DD, Phelps EA (1995) Impaired fear conditioning following unilateral temporal lobectomy in humans. J Neurosci 15:6846-6855.

Lang PJ, Greenwald MK, Bradley MM, Hamm AO (1993) Looking at pictures: affective, facial, visceral, and behavioral reactions. Psychophysiology 30:261-273.

LeDoux JE (1996) The emotional brain. New York: Simon and Schuster.

Lipp OV, Sheridan J, Siddle DAT (1994) Human blink startle during aversive and nonaversive Pavlovian conditioning. J Exp Psychol Anim Behav Process 20:380-389.

Lovibond PF, Shanks DR (2002) The role of awareness in Pavlovian conditioning: empirical evidence and theoretical implications. J Exp Psychol Anim Behav Process 28:3-26.

Lundqvist D, Flykt A, Öhman A (1998) The Karolinska Directed Emotional Faces (KDEF) [CD-ROM]. Stockholm: Karolinska Institutet.

Lykken DT, Venables PH (1971) Direct measurement of skin conductance: a proposal for standardization. Psychophysiology 8:656-672.

Maren S (2001) Neurobiology of Pavlovian fear conditioning. Annu Rev Neurosci 24:897-931.

Morris JS, Öhman A, Dolan RJ (1998) Conscious and unconscious emotional learning in the human amygdala. Nature 393:467-470.
Öhman A, Mineka S (2001) Fears, phobias, and preparedness: toward an evolved module of fear and fear learning. Psychol Rev 108:483-522.

Öhman A, Hamm A, Hugdahl K (2000) Cognition and the autonomic nervous system: orienting, anticipation, and conditioning. In: Handbook of psychophysiology, Ed 2 (Cacioppo JT, Tassinary LG, Berntson GG, eds), pp 533-575. Cambridge, UK: Cambridge UP.

Penfild W, Baldwin M (1952) Temporal lobe seizures and technique of subtotal lobectomy. Ann Surg 136:625-634.

Peper M, Karcher S, Wohlfarth R, Reinshagen G, LeDoux JE (2001) Aversive learning in patients with unilateral lesions of the amygdala and hippocampus. Biol Psychol 58:1-23.

Phelps EA, O’Connor KJ, Gatenby JC, Gore JC, Grillon C, Davis M (2001) Activation of the left amygdala to a cognitive representation of fear. Nat Neurosci 4:437-441.

Prokasy WF, Kumpfer KL (1973) Classical conditioning. In: Electrodermal activity in psychophysiological research (Prokasy WF, Raskin DC, eds), pp 157-202. New York: Academic.

Rescorla RA (1988) Pavlovian conditioning: it's not what you think it is. Am Psychol 43:151-160.

Squire LR, Knowlton BJ (2000) The medial temporal lobe, the hippocampus, and the memory systems of the brain. In: The new cognitive neuroscience (Gazzaniga MS, ed), pp 765-779. Cambridge, MA: MIT.

Venables PH, Christie MJ (1980) Electrodermal activity. In: Techniques in psychophysiology (Martin I, Venables PH, eds), pp 3-67. Chichester, UK: Wiley.

Walker DL, Davis M (2002) The role of amygdala glutamate receptors in fear learning, fear-potentiated startle, and extinction. Pharmacol Biochem Behav 71:379-392.

Wieser HG, Yasargil MG (1982) Selective amygdalahippocampectomy as a surgical treatment of mesobasial limbic epilepsy. Surg Neurol 17:445457. 